УДК 621.311

\title{
МЕТОДИКА МОДЕЛИРОВАНИЯ РЕГИОНАЛЬНО ОБОСОБЛЕННОГО ЭЛЕКТРОТЕХНИЧЕСКОГО КОМПЛЕКСА
}

\author{
Архипова Ольга Владимировна', \\ arkh82@mail.ru
}

\author{
Ковалев Владимир Захарович', \\ vz_kovalev@mail.ru
}

\author{
Хамитов Рустам Нуриманович², \\ apple_27@mail.ru \\ 1 Югорский государственный университет, \\ Россия, 628012, г. Ханты-Мансийск, ул. Чехова, 16. \\ 2 Омский государственный технический университет, \\ Россия, 644050, г. Омск, пр. Мира, 11.
}

\begin{abstract}
Актуальность исследования обусловлена необходимостью устранения разрыва между существующими подходами к моделированию изолированных систем электроснабжения и необходимостью учета динамических процессов в их физически разнородных подсистемах, образующих совокупность, при наличии слабых связей и слабых взаимодействий между единичными системами.

Цель исследования: сконструировать проблемно-ориентированный численный метод, непосредственно применимый к математическим моделям совокупности изолированных систем электроснабжения и существенно превосходящий по критерию «время счета - точность счета» универсальные численные методы.

Объект: электротехнический комплекс, состоящий из совокупности изолированных систем электроснабжения, объединенных слабыми связями и слабыми взаимодействиями.

Методы. При анализе формы и структуры исходной математической модели совокупности электротехнических комплексов, состоящих из изолированных систем электроснабжения, объединенных слабыми связями и слабыми взаимодействиями, и при конструировании проблемно-ориентированного численного метода использованы: энергетический подход к построению математических моделей электротехнических комплексов и систем, теория численных методов решения жестких систем дифференциальных уравнений, корректные допущения при составлении математических моделей и компьютерное моделирование.

Результаты. Введено в рассмотрение понятие «Регионально обособленный электротехнический комплекс», и выявлены его характерные признаки. Показана возможность создания новых алгоритмов управления «Регионально обособленным электротехническим комплексом», в целях снижения возможного экологического ущерба и средней расчётной себестоимости производства электроэнергии электростанциями, входящими в «Регионально обособленный электротехнический комплекс». Выявлена исходная структура математической модели «Регионально обособленного электротехнического комплекса». Показано принципиальное отличие структуры данной модели от модели в нормальной форме Коши. Обосновано принципиальное наличие свойств жесткости и сверхжесткости у анализируемого класса моделей. Сконструирован и реализован в программе ВКМ-3 проблемно ориентированный трехшаговый численный метод, применимый к исходной математической модели «Регионально обособленного электротехнического комплекса». Проведен численный эксперимент, показавший, на идеализированных математических моделях «Регионально обособленного электротехнического комплекса», существенное преимущество программы ВКМ3, по критерию «время счета - точность счета» в области применимости.
\end{abstract}

\section{Ключевые слова:}

Регионально обособленный электротехнический комплекс, возобновляемые источники энергии, оптимизация, численные методы, энергоэффективность.

\section{Введение}

Характер развития мировой экономики в значительной мере обусловлен увеличением объемов потребления электрической энергии и экспансией сырьевых отраслей промышленности в области, удаленные от централизованного электроснабжения [1]. Соответственно возникает задача оптимального проектирования совокупностей изолированных систем электроснабжения для децентрализованных зон электроснабжения.

В первую очередь сюда относятся районы Крайнего Севера, Восточной Сибири, Дальнего Востока. Здесь добывается более $75 \%$ российской нефти, более $90 \%$ российского газа. Большая часть этих территорий находится в условиях децентрализо- ванного энергоснабжения, где осуществляется жизнедеятельность более чем в 70 городах, 360 поселках и 1400 малых населенных пунктах [1, 2].

Изолированные системы электроснабжения (ИСЭ) строятся, как правило, на базе дизельных электростанций (ДЭС). Рассматриваемый класс ИСЭ характеризуется: отсутствием электрической связи с крупными энергосистемами, отсутствием электрической связи между отдельными ИСЭ, сложной логистикой доставки топлива, запасных частей и других расходных материалов. Сезонность поставок, их многоэтапность: железная дорога, речной флот, «зимники», вертолеты, автомобильный транспорт - приводят к высокой себестоимости вырабатываемой электроэнергии из-за по- 
вышения транспортной составляющей стоимости топлива. Так, в прибрежной Арктической зоне цена топлива возрастает от $1,2-1,3$ раза при использовании автотранспорта до 2-3 раз при использовании авиадоставок [3, 4]. Как следствие, в некоторых поселениях рассматриваемых районов себестоимость одного киловатт-часа электроэнергии зачастую превышает 25-40 р. и может доходить до 150 р. и выше [4].

В то же время регионы расположения рассматриваемого класса ИСЭ, как правило, обладают значительными ресурсами возобновляемых источников энергии (ВИЭ): ветровая энергия, гидроэнергия малых рек, геотермальная энергия, солнечная энергия и другие. При этом климатические и логистические различия отдельных зон присутствия ИСЭ определяют зачастую противоположные требования к выбору применяемого типа ВИЭ или даже к отказу от использования.

Следующая «исторически сложившаяся» особенность данных ИСЭ - обилие в одном регионе используемых типоразмеров основного оборудования и их изготовителей. Например, по данным [5], на семь ИСЭ Ленинского района Якутии приходится семь (!) типоразмеров дизель-электрических установок (ДЭУ) нескольких заводов изготовителей. При этом у пяти из семи ИСЭ фактические ежегодные выработки электроэнергии практически равны (табл. 1). Что говорит о нерациональном подходе к типизации оборудования.

Неоспоримо влияние дизельной генерации электроэнергии на экологию региона размещения и «в то же время стремление каждого предприятия самостоятельно решить означенные выше проблемы, что в целом снижает эффективность инвестиционных вложений в энергоэкологические мероприятия» [6].

Данные обстоятельства вызывают дополнительные расходы на логистику, складские запасы, обслуживание и ремонт. Как следствие - увеличенная нагрузка на бюджеты регионов и корпораций, снижение показателей энергоэффективности, негативное влияние на экологические параметры, применительно к региону размещения ИСЭ [7].

Отметим, как одно из новых направлений развития ИСЭ, применение накопителей энергии: гравитационных, кинетических, на основе сжатого воздуха, электрохимических и других [8]. Промышленное применение таких устройств в составе гибридных (сочетающих несколько физически разнородных принципов преобразования энергии) автономных электростанций пока единично.

Существует ряд специфических электротехнических комплексов, составляющих ИСЭ, для которых динамические режимы работы являются основными и обусловлены реализуемым технологическим процессом. Характерный пример такого потребителя - электротехнический комплекс буровой установки [9]. Следствие из указанного обстоятельства - либо оптимизация РОЭТК (как совокупности ИСЭ) на принципах декомпозиции си- стемы с последующей ее обратной сборкой (как принято в настоящее время) с естественной потерей степени адекватности, либо конструирование методов, работающих с приемлемой характеристикой «время счета - точность счета». Что важно для последующей оптимизации всей РОЭТК с учетом слабых связей.

В то же время современные методики оптимизации структуры и состава ИСЭ фактически ориентированы на оптимизацию экологоэнергетических показателей единичной ИСЭ, без строгого учета взаимного влияния принимаемых проектных решений по отдельным ИСЭ. Основой таких методик служат усредненные многолетние статистические данные [5, 10-17].

Возникает задача оптимального сочетания надежной, хорошо освоенной технологии дизельной генерации электроэнергии с развивающимися технологиями использования ВИЭ $[18,19]$. В настоящее время комбинированное использование этих видов энергии носит эпизодический и исследовательский характер, хотя не подлежит сомнению важная социально-экономическая роль таких подходов [3, 20, 21].

Очевидно, что утверждение 23 января 2015 г. Правительством РФ Постановления № 47 «0 внесении изменений в некоторые акты Правительства РФ по вопросам стимулирования использования возобновляемых источников энергии на розничных рынках электроэнергии" окажет дополнительное стимулирующее воздействие на увеличение доли ВИЭ в энергобалансах ИСЭ [22].

Указанные особенности и противоречия для своего разрешения требуют создания теории и методики проектирования оптимальных, в некотором смысле, совокупностей ИСЭ рассматриваемого класса с использованием ВИЭ.

\section{Теория}

Введем в рассмотрение следующее понятие: «Регионально обособленный электротехнический комплекс (РОЭТК)»- обособленная совокупность изолированных систем электроснабжения, характеризующаяся отсутствием электрической связи между отдельными ИСЭ, при одновременном наличии «слабых связей» и «слабых взаимодействий» между ИСЭ, входящими в данную совокупность. Причем «слабые связи» и «слабые взаимодействия» влияют как на интегральные показатели указанной совокупности (энергоэффективность, уровень экологичности производства электроэнергии, себестоимость производства электроэнергии), так и на структуру и принципы построения и функционирования каждой из изолированных систем электроснабжения данной совокупности на протяжении всего жизненного цикла.

Отметим:

термины «слабые связи» и «слабые взаимодействия» в данном контексте соответствуют смыслу определений Б.И. Кудрина и В.К. Лозенко и коррелируются с понятием «бизнес-связи» [7]; 
предложенная в данной работе формулировка понятия РОЭТК является логическим развитием работ [7, 21].

Сформулированное выше понятие РОЭТК обусловливает жесткую необходимость оптимизации всей совокупности ИСЭ, входящих в РОЭТК. В качестве критерия оптимизации может выступать энергоэффективность в сочетании с требованиями типизации, экологичности, надежности $[1,3,5,6$, $16,23,24]$. Обобщающими в данном случае становятся LCOE, SLCOE, LACE - «критерии, основанные на оценке средней расчётной себестоимости производства электроэнергии на протяжении всего жизненного цикла электростанции (включая все возможные инвестиции, затраты и доходы)» [25].

Таблица 1. Типоразмер ДЭС совокупности ИСЭ Ленинского ра йона Якутии (по данным [5])

Table 1. Typical size of a diesel power plant (DPP) of the isolated electrical power supply system (IEPSS) complex of Leninsky district of Yakutia (according to [5])

\begin{tabular}{|c|c|c|c|c|c|c|c|c|c|}
\hline \begin{tabular}{|c|} 
Мощность ди- \\
зельной электро- \\
генераторной \\
установки (ДЭУ), \\
кВт \\
Capacity of diesel \\
electricgenerating \\
set (DES), kW
\end{tabular} & 200 & 100 & 75 & 60 & 30 & 16 & 8 & 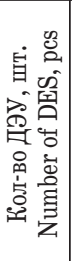 & 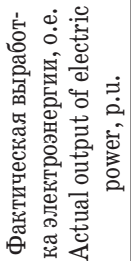 \\
\hline \begin{tabular}{c|} 
c. Чамча \\
vil. Chamcha
\end{tabular} & - & 2 & - & 1 & - & - & - & 3 & 1,2 \\
\hline $\begin{array}{c}\text { c. Xaмpa } \\
\text { vil. Khamra }\end{array}$ & - & 1 & 1 & 1 & 1 & - & - & 4 & 1,0 \\
\hline $\begin{array}{l}\text { c. Натора } \\
\text { vil. Natora }\end{array}$ & 1 & 2 & - & - & - & - & - & 3 & 0,96 \\
\hline $\begin{array}{c}\text { c. Турукта } \\
\text { vil. Turukta }\end{array}$ & - & 1 & 1 & 2 & 1 & - & - & 5 & 0,82 \\
\hline $\begin{array}{c}\text { c. Толон } \\
\text { vil. Tolon }\end{array}$ & - & 1 & - & 2 & 1 & - & - & 4 & 0,80 \\
\hline $\begin{array}{l}\text { c. Иннялы } \\
\text { vil. Innyaly }\end{array}$ & - & - & - & - & 2 & 1 & - & 3 & 0,42 \\
\hline $\begin{array}{c}\text { c. Алысардах } \\
\text { vil. Alysardakh }\end{array}$ & - & - & - & - & - & - & 1 & 2 & 0,045 \\
\hline
\end{tabular}

Соответственно первоочередными становятся задачи:

- выявление исходной структуры математической модели совокупности энергопреобразующих элементов РОЭТК, с учетом «слабых связей» и «слабых взаимодействий»;

- конструирование проблемно-ориентированного численного метода, применимого к исходной математической модели РОЭТК;

- проведение численного эксперимента по сравнению программных реализаций численных методов извлечения информации из исходной математической модели РОЭТК;

- построение системы географических, климатических, административных, корпоративных или иных критериев включения отдельных ИСЭ в РОЭТК;

- построение системы географических, климатических, административных, корпоративных, экономических или иных критериев включения конкретного вида/видов ВИЭ в состав отдельной ИСЭ входящей в РОЭТК;

- построение критерия/критериев оптимальности РОЭТК;

- построение методики оптимизации РОЭТК.

Отметим, что предметом данной статьи являются первые три из указанных задач. Возможные пути решение остальных задач будут рассмотрены в последующих публикациях.

Энергетический подход к математическому моделированию электротехнических комплексов и систем, развиваемый в работе [26] и базирующийся на формализме Лагранжа, с неукоснительностью приводит к построению исходных математических моделей основных взаимодействующих физически разнородных энергопреобразующих элементов РОЭТК в следующем виде:

$$
\begin{aligned}
\frac{d U_{*}^{T}}{d t} & =f\left(Q_{\Sigma}, Q_{b}, t\right), \\
U_{*}^{T} & =U_{*}^{T}\left(Q_{\Sigma}, Q_{b}\right),
\end{aligned}
$$

где $Q_{\Sigma}$ и $Q_{b}$ - векторы обобщенных координат и обобщенных скоростей.

Идентификацию параметров электромеханических и других компонент РОЭТК, входящих в данную модель, предлагается проводить по методикам, синтезированным на основании работ [27, 28].

Исходная форма уравнений $(1),(2)$ для своего решения требует подстановки алгебраических уравнений (2) в уравнения (1) и соответствующего построения матрицы Якоби:

$$
A\left(Q, Q_{b}\right)=\partial U_{*}^{T} / \partial Q_{\Sigma} .
$$

Далее для перехода к модели, представленной нормальной формой Коши, необходима трудоемкая (до 90 \% времени счета) операция обращения матрицы Якоби (3). Как следствие возникает необходимость конструирования специализированного численного метода, применимого к исходным уравнениям (1), (2).

Физическая разнородность взаимодействующих в РОЭТК компонент (электрические цепи, магнитные цепи, механические цепи, химические элементы, ...) приводит к наличию в математической модели различающихся на порядки постоянных времени, так называемая «жесткость» и «сверхжесткость» модели $[26,29]$. Численно это явление характеризуется коэффициентом жесткости $k_{ж}$ :

$$
k_{\text {ж }}=\max _{1 \leq k \leq n} \tau_{k} / \min _{1 \leq m \leq n} \tau_{m},
$$

где $n$ - размерность системы уравнений; $\tau$ - постоянная времени системы.

Принято считать математическую модель жесткой при $k_{\text {ж }}>100$.

Указанное обстоятельство, в свою очередь, накладывает требования на вычислительную устойчивость применяемого для решения системы уравнений (1), (2) численного метода [26, 29, 30]. 
В данном направлении разработан непосредственно применимый к модели (1), (2) численный трехшаговый метод вида:

$$
\begin{aligned}
& Q_{\text {SUM }(n+3)}(t+3 h)=Q_{\text {SUM }(n)}(t)+ \\
& +G\left[\begin{array}{l}
\left(A \psi, A_{f}, h, a_{\text {неяв }}\right)_{(n+k)}^{m} ; \beta_{\text {0неяв }} ; \beta_{1 \text { 1еяв }} ; \\
\beta_{\text {2неяв }} ; \beta_{\text {Знеяв }} ; a_{\text {неяв }} ; h ; f_{n} ; f_{n+1} ; f_{n+2} ; f_{n+3}
\end{array}\right],
\end{aligned}
$$

где $G$ - функционал; $Q_{S U M(n)}-$ суммарный вектор $Q_{\Sigma}$ и $Q_{b}$ в момент времени $t ; h$ - шаг интегрирования; $a_{\text {неяв }}$ и $\beta_{3 \text { неяв }}-$ свободные параметры метода;

$$
A_{\psi}=\frac{\partial U\left(Q_{S U M}\right)}{\partial Q_{S U M}} ; A_{f}=\frac{\partial f_{*}^{m}\left(Q_{S U M}, t\right)}{\partial Q_{S U M}} .
$$

Предложенный метод является проблемно-ориентированным развитием группы линейных многошаговых методов [31], обладает свойством А-устойчивости и третьим порядком точности, в смысле определений работ G. Hall, J.M. Watt, E. Hairer, G. Wanner [29, 31].

\section{Результаты вычислительных экспериментов}

Сравнительная оценка вычислительных свойств метода (3) производилась на наборе тестовых математических моделей, имеющем точное решение и содержащем как модели в нормальной форме Коши, так и модели в форме (1), (2). Структура уравнений данных моделей синтезирована так, чтобы соответствовать структуре математической модели ДЭУ с асинхронной машиной в режиме генератора (АГ), при допущении о ненасыщенности магнитопровода (тест 1). Набор переменных коэффициентов в тестовой модели позволяет задавать вычислительные характеристики, соответствующие различными схемами построения ИСЭ при использовании ВИЭ [8, 10, 13-15]. Тест 2 учитывает в модели ИСЭ наличие накопителя энергии НЭ [32, 33], дополнительно к ДЭУ с АГ. Тест 3 соответствует структуре модели ИСЭ в составе: ДЭУ, $\mathrm{AГ,} \mathrm{НЭ} \mathrm{и} \mathrm{ветрогенераторной} \mathrm{установки} \mathrm{(ВЭУ)} \mathrm{[16,}$ 34]. Тесты 4, 5 эквивалентируют вычислительные свойства математической модели гибридной ИСЭ в составе: ДЭУ, АГ, НЭ, ВЭУ, фотоэлектрическая энергоустановка (ФЭУ) и преобразователь напряжения (AC-DC-AC) соответственно [35-37]. Тесты 6, 7 - математические модели в нормальной форме Коши [29].

Характерное для математических моделей РОЭТК свойство жесткости (4) моделируется в те-

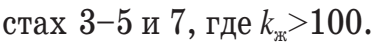

Соответственно, требуются жесткоустойчивые численные методы извлечения информации из математических моделей. Для сравнительного тестирования принята версия методов Гира [30], реализованная в программе DIFSUB [38].

Предлагаемый проблемно-ориентированный численный метод (5) реализован в программе BKM-3. Здесь использованы принципы изменения шага интегрирования и оценки локальной и глобальной погрешностей, реализованные в программе BKM-2 [26] и коррелирующиеся с принципами построения вычислительного процесса в программe DIFSUB.

Оценивалось время счета при равных заданных относительных погрешностях (критерий «время счета - точность счета»). Время счета программой BKM-2 принималось за единицу. Результаты сравнительного тестирования для случая заданной относительной погрешности $e<0,01$ приведены в табл. 2.

Анализ результатов вычислительного эксперимента показал:

- программа ВKM-3, реализующая формулу численного метода (5), эффективна на математических моделях, представленных в форме (1), (2) (задачи 1-5, табл. 2);

- преимущество программы BKM-3 по критерию «время счета - точность счета» зависит от коэффициента жесткости модели, ее размерности, допустимой погрешности расчета;

- программа BKM-3 на задачах в нормальной форме Коши (задачи 6,7 , табл. 2) преимуществ не имеет.

Таким образом, область применимости предложенного метода (5), реализованного в программе BKM-3, можно определить как математические модели РОЭТК в форме уравнений (1), (2).

\section{Обсуждение результатов}

Выявленный существенный признак РОЭТК наличие «слабых связей» и «слабых взаимодействий», определяет особенности и отличия каждого регионально обособленного электротехнического комплекса в целом. Индивидуальные характеристики РОЭТК формируются значительной территориальной удаленностью от центра энергетических нагрузок ИЭС, входящих в РОЭТК. Климатические и логистические различия отдельных $30 \mathrm{H}$ присутствия РОЭТК определяют зачастую противоположные требования к выбору типа ВИЭ внутри РОЭТК.

Таблица 2. Относительное вреля счёта тестовых моделей РОЭТК при допустимой погрешности е $<0,01$

Table 2. Relative time of regionally isolated electrotechnical com-

\begin{tabular}{|c|c|c|c|c|}
\hline $\begin{array}{c}\text { Тестовая модель } \\
\text { РОЭТК (тест) } \\
\text { RIEC test model } \\
\text { (test) }\end{array}$ & $\begin{array}{c}\text { Коэффициент жестко- } \\
\text { сти модели РОЭТК, } k_{\text {ж }} \\
\text { Stiffness factor of RIEC } \\
\text { model, } k_{s}\end{array}$ & DIFSB & BKM-2 & BKM-3 \\
\hline 1 & 3 & 11 & \multirow{7}{*}{1} & 0,41 \\
\hline 2 & 30 & 12 & & 0,43 \\
\hline 3 & 300 & 12 & & 0,33 \\
\hline 4 & 1600 & 10 & & 0,36 \\
\hline 5 & 3000 & 9 & & 0,28 \\
\hline 6 & 30 & 0,49 & & 0,75 \\
\hline 7 & 3000 & 0,63 & & 0,71 \\
\hline
\end{tabular}
plex (RIEC) test models calculation with allowable error $e<0,01$

Учет указанных обстоятельств в единой математической модели РОЭТК в целях последующей оценки экологических параметров и энергоэффективности всего РОЭТК в целом приводит к появле- 
нию свойств жесткости и сверхжесткости математической модели (1), (2). При этом математическая модель РОЭТК имеет исходную форму, отличную от нормальной формы Коши. Условия сводимости модели (1), (2) к нормальной форме Коши, степень снижения ее адекватности при этой процедуре требует отдельного исследования.

Предложенный численный метод (5) и его программная реализация ВKM-3, в силу «узкой» специализации на моделях вида (1), (2), имеет многократные преимущества перед универсальными программами и позволяет перейти к решению задач анализа динамических режимов работы гибридных электростанций с позиций управления сверхкраткосрочными прогнозами ресурсов ВИЭ и соответствующей оптимизации структуры РОЭТК на протяжении всего жизненного цикла.

\section{Выводы}

1. Введено в рассмотрение понятие «Регионально обособленный электротехнический комплекс

\section{СПИСОК ЛИТЕРАТУРЫ}

1. Lukutin B.V. Simulation and optimization of wind and diesel power supply systems // IOP Conference Series: Materials Science and Engineering. - Tomsk, 2017. - P. 012090.

2. Елистратов В.В Автономное энергоснабжение энергокомплексами на базе возобновляемых источников энергии // Сантехника, отопление, кондиционирование. - 2016. - № 3 (171). C. $72-75$.

3. Елистратов В.В Энергетический, экологический и социальноэкономический аспекты в энергоснабжении северных и арктических территорий РФ // Экологический вестник России. 2017. - № 11. - С. 30-35.

4. Минин В.А., Рожкова А.А. Оценка эффективности внедрения ветроэнергетических установок на дизельных электростанциях в арктической зоне РФ // Труды Кольского научного центра РАН. - 2017. - № 1-14 (8). - С. 93-99.

5. Карамов Д.Н. Оптимизация состава оборудования автономных энергокомплексов, использующих возобновляемые источники и накопители энергии: дис.... канд. техн. наук. - Иркутск, 2016. -152 c.

6. Балабанов М.С., Бабошкина С.В., Хамитов Р.Н. Экологические аспекты в энергосберегающей политике на этапе создания в России интеллектуальных энергосистем с активноадаптивной сетью // Известия Томского политехнического университета. Инжиниринг георесурсов. - 2015. - Т. 326. - № 11. C. $141-152$.

7. Ковалев В.З., Архипова О.В. Энергетические аспекты регионально обособленного электротехнического комплекса // Вестник Югорского государственного университета. - 2015. № S2 (37). - C. 217-218.

8. Тарасенко А.Б., Попель 0.С Подходы к оценке и примеры применимости накопителей электрической энергии для различных приложений // Возобновляемая энергетика XXI век: Энергетическая и экономическая эффективность: Материалы Международного конгресса REENCON-XXI. - M., 2016. C. $243-249$.

9. Оптимизация загрузки дизель-генераторов автономной системы электроснабжения куста буровых установок // Официальный сайт Группы Компаний «ДИАЛ». URL: http://compensation.ru/articles/optimizatsiya-zagruzki-dizel-generatorov-avtonomnoy-sistemy-elektrosnabzheniya-kusta-burovykh-ustano (дата обращения 29.11.2018).
(РОЭТК)» - обособленная совокупность изолированных систем электроснабжения.

2. Выявлена исходная структура математических моделей основных энергопреобразующих элементов РОЭТК, с учетом характерных «слабых связей» и «слабых влияний». Показано принципиальное отличие структуры данной модели от нормальной формы Коши.

3. Сконструирован и реализован в программе BKM-3 специализированный трехшаговый численный метод, применимый к исходной математической модели РОЭТК.

4. Проведен численный эксперимент, показавший на математических моделях РОЭТК существенное преимущество программы BKM-3, по критерию «время счета - точность счета», в области применимости.

Исследование выполнено при финансовой поддержке РФФИ и Правительства ХМАО-Югры в рамках научного проекта № 18-47-860017.

10. Xiaonan Wang, Ahmet Palazogluy, Nael H. El-Farra. Operation of Residential Hybrid Renewable Energy Systems: Integrating Forecasting, Optimization and Demand Response // 2014 American Control Conference (ACC). - Portland, Oregon, USA, June 4-6, 2014. - P. 5043-5048.

11. Марченко 0.В., Соломин С.В. Анализ совместного использования энергии солнца и ветра в системах автономного электроснабжения // Промышленная энергетика. - 2016. - № 9. C. $39-43$.

12. Minna Ranjeva, Anil K. Kulkarni. Design Optimization of a Hybrid, Small, Decentralized Power Plant for Remote/Rural Areas // Energy Procedia. - 2012. - V. 20. - P. 258-270.

13. Суслов К.В. Развитие систем электроснабжения изолированных территорий России с использованием возобновляемых источников энергии // Вестник Иркутского государственного технического университета. - 2017. - Т. 21. - № 5 (124). - С. 131-142.

14. Atiqur Rahman M.M., Al Awami A.T., Rahim A.H.M.A. HydroPV-wind-battery-diesel based stand-alone hybrid power system // International Conference on Electrical Engineering and Information \& Communication Technology (ICEEICT). - Dhaka, 2014. P. $1-6$.

15. Experimental analysis of a solar PV/diesel hybrid system without storage: Focus on its dynamic behavior / D. Yamegueu, Y. Azoumah, X. Py, H. Kottin // International Journal of Electrical Power \& Energy Systems. - 2013. - V. 44. - P. 267-274.

16. Kovalev V.Z., Arkhipova 0.V. Structural optimization technology for fleet of wind-diesel power plants // Petroleum Engineering: scientific and technical journal. - 2014. - V. 12. - № 4. P. 119-124.

17. Технико-экономический анализ применения ветро-дизельных электростанций для электроснабжения энергоудаленных поселений / Е.Н. Соснина, А.В. Шалухо, И.А. Липужин, Т.А. Александрова // Труды НГТУ им. Р.Е. Алексеева. 2016. - № 1. - C. 65-72.

18. Dufo-López R., Bernal-Agustín J.L. Multi-objective optimization minimizing cost and life cycle emissions of stand-alone PV-wind-diesel systems with batteries storage // Applied Energy. - 2011. - V. 88. - P. 4033-4041.

19. Dekker J., Nthontho M., Chowdhury S. Economic analysis of $\mathrm{PV} /$ diesel hybrid power systems in different climatic zones of South Africa / International Journal of Electrical Power \& Energy Systems. - 2012. - V. 40. - № 1. - P. 104-112. 
20. Lahimer A.A., Alghoul M.A., Yousif Fadhil. Research and development aspects on decentralized electrification options for rural household // Renewable and Sustainable Energy Reviews. 2013. - V. 24. - P. 314-324.

21. Архипова 0.В. Принципы оптимизации электроснабжения населенных пунктов крайнего севера на базе ветродизельных комплексов // Вестник Югорского государственного университета. - 2015. - № S2 (37). - C. 204-206.

22. 0 внесении изменений в некоторые акты Правительства Российской Федерации по вопросам стимулирования использования возобновляемых источников энергии на розничных рынках электрической энергии: Постановление Правительства РФ от 23.01.2015 № 47 // Собрание законодательства РФ. 2005. - № 5. - Ст. 827.

23. Hopulele E., Gavrilas M., Atanasoae P. Optimal design of a hybrid distributed generation system // 49th International Universities Power Engineering Conference (UPEC). - Cluj-Napoca, 2014. - P. 1-6.

24. Decentralized energy generation for end-use applications: Economic, social and environmental benefits assessment / D.0. Akinyele, R.K. Rayudu, N.K.C. Nair, B. Chakrabarti // Innovative Smart Grid Technologies - Asia (ISGT Asia), IEEE. - Kuala Lumpur, 2014. - P. 84-89.

25. Черняховская Ю.В. Эволюция методологических подходов к оценке стоимости электроэнергии. Анализ зарубежного опыта // Вестник Ивановского государственного энергетического университета. - 2016. - № 4. - С. 56-68.

26. Ковалев В.З., Мальгин Г.В., Архипова 0.В. Математическое моделирование электротехнических комплексов нефтегазодобычи в задачах энергосбережения: монография. - Ханты-Мансийск: Департамент образования и науки ХМАО-Югры, Югорский государственный университет, 2008. - 222 с.

27. Боловин Е.В., Глазырин А.С. Метод идентификации параметров погружных асинхронных электродвигателей установок электроприводных центробежных насосов для добычи нефти // Известия Томского политехнического университета. Инжиниринг георесурсов. - 2017. - Т. 328. - № 1. - С. 123-131.

28. Ковалев В.З., Щербаков А.Г. Информационная система идентификации параметров математических моделей электротех- нических комплексов // Системы управления и информационные технологии. - 2009. - Т. 35. - № 1. - С. 57-59.

29. Hairer E., Wanner G. Solving Ordinary Differential Equations II: Stiff and Differential-Algebraic Problems // Springer Series in Computational Mathematics. - 1996. - V. 14. - 614 p.

30. Гридин В.Н., Рыжов Н.Г., Анисимов В.И., Абухазим М.М. Методы повышения эффективности процессов моделирования динамических режимов нелинейных систем // Информационные технологии. - 2017. - Т. 23. - № 11. - С. 796-802.

31. Hall G. Modern Numerical Methods for Ordinary Differential Equations / ed. by G. Hall, J.M. Watt. - Oxford, UK: Clarendon Press, 1976. $-312 \mathrm{p}$.

32. Tremblay 0., Dessaint L. Experimental validation of a battery dynamic model for EV application // World Electric Vehicle Journal. - 2009. - № 3. - P. 1-10.

33. Thomachan A. Kattakayam, Srinivasan K. Lead acid batteries in solar refrigeration systems // Renewable Energy. - 2004. V. 29. - P. 1243-1250.

34. Musgrove P. Wind Energy. - Cambridge: Cambridge university press, 2010. $-323 \mathrm{p}$.

35. Salas V., Olias E. Overview of the state of technique for PV inverters used in low voltage gridconnected PV systems: Inverters above 10kW // Renewable and Sustainable Energy Reviews. - 2011. V. 15. - P. 1250-1257.

36. Hassaine L., Olias E., Quintero J. Overview of power inverter topologies and control structures for grid connected photovoltaic systems / Renewable and Sustainable Energy Reviews. - 2014. V. 30. - P. 796-807.

37. Levelized cost of electricity renewable energy technologies / C. Kost, S Shammugam., V. Jülch, H.-T. Nguyen, T. Schlegl. Freiburg: Fraunhofer institute for solar energy systems ISE, 2018. - $34 \mathrm{p}$.

38. Gear C.W. The Algorithm 407: DIFSUB for solution of ordinary differential equations // Communications of the ACM. - 1971. V. 14. - № 3. - P. 185-190.

Поступила 17.12.2018 г.

\section{Информация об авторах}

Apxunова O.B., старший преподаватель кафедры энергетики Югорского государственного университета.

Ковалев В.З., доктор технических наук, профессор, заведующий кафедрой энергетики Югорского государственного университета.

Хамитов P.H., доктор технических наук, профессор кафедры электрической техники Омского государственного технического университета. 
UDC 621.311

\title{
METHODOLOGY OF MODELING REGIONALLY ISOLATED ELECTROTECHNICAL COMPLEX
}

\author{
Olga V. Arkhipova', \\ arkh82@mail.ru
}

\author{
Vladimir Z. Kovalev', \\ vz_kovalev@mail.ru
}

\author{
Rustam N. Khamitov' \\ apple_27@mail.ru \\ 1 Yugra State University, \\ 16, Chekhov Street, Khanty-Mansiysk, 628012, Russia. \\ 2 Omsk State Technical University, \\ 11, Mira Avenue, Omsk, 644050, Russia.
}

The relevance of the research is caused by the need to bridge the gap between the existing approaches to modeling isolated systems of power supply considering dynamic processes in their physically diverse subsystems, in the presence of weak communications and weak interactions between single systems.

The aim of the research is to design the problem-oriented numerical method directly applicable to initial mathematical models of the isolated systems of power supply, and having advantage by criterion "counting duration - account accuracy» in relation to universal numerical methods.

Object: electrotechnical complex consisting of set of the isolated systems of power supply united by weak communications and weak interactions.

Methods. When analyzing a form and structure of initial mathematical model of set of the electrotechnical complexes consisting of the isolated systems of power supply united by weak communications and weak interactions, and when designing a problem-oriented numerical method the authors have used: power approach to mathematical modeling of electrotechnical complexes and systems, provision of theoretical bases of electrical equipment, theory of numerical methods of solution of rigid systems of the differential equations, correct assumptions by drawing up mathematical models and computer modeling.

Results. The paper introduces the concept «Regionally isolated electrotechnical complex» and the authors determine its characteristic signs. The article considers the possibility of developing new algorithms for controlling the "Regionally isolated electrotechnical complex» to decrease the possible ecological damage and average settlement cost of electric power production by the power plants included in "Regionally isolated electrotechnical complex». The authors determined the initial structure of the mathematical model of "Regionally isolated electrotechnical complex». The paper demonstrates the fundamental difference between the structure of the given model and the model in a normal form of Cauchy. Basic presence of properties of rigidity and superrigidity at the analyzed class of models is given. The authors designed and realized in the BKM-3 program the focused three-step numerical method applicable to the initial mathematical model of "Regionally isolated electrotechnical complex». The authors carried out the numerical experiment which shown the essential advantage of the BKM-3 program, by criterion "counting duration - account accuracy» in the field of applicability, on the idealized mathematical models of "Regionally isolated electrotechnical complex».

\section{Key words:}

Regionally isolated electrotechnical complex, renewable energy sources, optimization, numerical methods, energy efficiency.

The research was financially supported by the RFBR and the Government of the Khanty-Mansiysk Autonomous region-Yugra within the scientific project no. 18-47-860017.

\section{REFERENCES}

1. Lukutin B.V. Simulation and optimization of wind and diesel power supply systems. IOP Conference Series: Materials Science and Engineering. Tomsk, 2017. pp. 012090.

2. Elistratov V.V Autonomous power supply by power complexes based on renewable energy sources. Plumbing, heating, air conditioning, 2016, no. 3 (171), pp. 72-75. In Rus.

3. Elistratov V.V Energy, environmental and socio-economic aspects in the energy supply of the northern and Arctic territories of the Russian Federation. Ecological Bulletin of Russia, 2017, no. 11, pp. 30-35. In Rus.

4. Minin V.A., Rozhkova A.A. Evaluation of the effectiveness of introducing wind power plants on diesel power plants in the Arctic zone of the Russian Federation. Works of the Kola peninsula Scientific Center of the Russian Academy of Sciences, 2017, no. 1-14 (8), pp. 93-99. In Rus.

5. Karamov D.N. Optimizatsiya sostava oborudovaniya avtonom nykh energokompleksov, ispolzuyushchikh vozobnovlyaemye is tochniki i nakopiteli energii. Dis. Kand. nauk [Optimization of the equipment of autonomous power complexes using renewable sources and energy storage. Cand. Diss.]. Irkutsk, 2016. 152 p.

6. Balabanov M.S., Baboshkina S.V., Khamitov R.N. Environmental aspects in the energy-saving policy at the stage of development of intelligent energy systems with an actively adaptive network in Russia. Bulletin of the Tomsk Polytechnic University. Geo Assets Engineering, 2015, vol. 326, no. 11, pp. 141-152. In Rus.

7. Kovalev V.Z., Arkhipova O.V. Energy aspects of a regionally isolated electrical complex. Bulletin of the Yugra State University, 2015, no. S2 (37), pp. 217-218. In Rus.

8. Tarasenko A.B., Popel O.S. Podkhody k otsenke i primery primenimosti nakopiteley elektricheskoy energii dlya razlichnyhk prilozheniy [Assessment approaches and examples of the applicability of electrical energy storage devices for various applications]. Vozobnovlyaemaya energetika XXI vek: Energeticheskaya i ekonomicheskaya effektionost. Materialy Mezhdunarodnogo kongressa REENCON-XXI [Renewable Energy XXI Century: Energy and Economic Efficiency. Proc. of the International Congress]. Moscow, 2016. pp. 243-249. 
9. Optimizatsiya zagruzki dizel-generatorov avtonomnoy sistemy elektrosnabzheniya kusta burovykh ustanovok [Optimization of loading diesel generators of an autonomous power supply system of a drilling rig bush]. DIAL Group of Companies. Available at: http://compensation.ru/articles/optimizatsiya-zagruzki-dizelgeneratorov-avtonomnoy-sistemy-elektrosnabzheniya-kusta-burovykh-ustano (accessed 29 November 2018).

10. Xiaonan Wang, Ahmet Palazogluy, Nael H. El-Farra. Operation of Residential Hybrid Renewable Energy Systems: Integrating Forecasting, Optimization and Demand Response. American Control Conference (ACC). Portland, Oregon, USA, June 4-6, 2014. pp. 5043-5048.

11. Marchenko 0.V., Solomin S.V. Analysis of the sharing of solar and wind energy in the systems of autonomous power supply. Industrial Energy, 2016, no. 9, pp. 39-43. In Rus.

12. Minna Ranjeva, Anil K. Kulkarni/ Design Optimization of a Hybrid, Small, Decentralized Power Plant for Remote/Rural Areas. Energy Procedia, 2012, vol. 20, pp. 258-270.

13. Suslov K.V. Development of power supply systems in isolated areas of Russia using renewable energy sources. Bulletin of Irkutsk State Technical University, 2017, vol. 21, no. 5 (124), pp. 131-142. In Rus.

14. Atiqur Rahman M.M., Al Awami A.T., Rahim A.H.M.A. Hydro-PVwind-battery-diesel based stand-alone hybrid power system. International Conference on Electrical Engineering and Information \& Communication Technology (ICEEICT). Dhaka, 2014. pp. 1-6.

15. Yamegueu D., Azoumah Y., Py X., Kottin H. Experimental analysis of a solar PV/diesel hybrid system without storage: Focus on its dynamic behavior. International Journal of Electrical Power \& Energy Systems, 2013, vol. 44, pp. 267-274.

16. Kovalev V.Z., Arkhipova 0.V. Structural optimization technology for fleet of wind-diesel power plants. Petroleum Engineering: scientific and technical journal, 2014, vol. 12, no. 4, pp. 119-124.

17. Sosnina E.N., Shaluxo A.V., Lipuzhin I.A., Aleksandrova T.A. Technical and economic analysis of the use of wind-diesel power plants for power supply of energy-remote settlements. Procee dings of the NSTU.R.E. Alekseeva, 2016, no. 1, pp. 65-72. In Rus.

18. Dufo-López R., Bernal-Agustín J.L. Multi-objective optimization minimizing cost and life cycle emissions of stand-alone PV-wind-diesel systems with batteries storage. Applied Energy, 2011, vol. 88, pp. 4033-4041.

19. Dekker J., Nthontho M., Chowdhury S. Economic analysis of PV/diesel hybrid power systems in different climatic zones of South Africa. International Journal of Electrical Power \& Energy Systems, 2012, vol. 40, no. 1, pp. 104-112.

20. Lahimer A.A., Alghoul M.A., Yousif Fadhil. Research and development aspects on decentralized electrification options for rural household. Renewable and Sustainable Energy Reviews, 2013, vol. 24, pp. 314-324.

21. Arkhipova 0.V. Principles of optimization of power supply of settlements of the far north on the basis of wind-diesel complexes. Bulletin of Yugra State University, 2015, no. S2 (37), pp. 204-206. In Rus.

22. Postanovlenie pravitelstva RF, 23.01.2015 № 47 «0 vnesenii izmeneny v nekotorye akty Pravitelstva Rossiyskoy Federatsii po voprosam stimulirovaniya ispolzovaniya vozobnovlyaemykh istochnikov energii na roznichnykh rynkakh elektricheskoy energii [On Amendments to Certain Acts of the Government of the Russian Federation on the Promotion of the Use of Renewable Energy Sources in Retail Electricity Markets: Resolution of the Government of the Russian Federation of January 23, 2015 no 47]. Collection of legislation of the Russian Federation, 2005, no. 5, art. 827.
23. Hopulele E., Gavrilas M., Atanasoae P. Optimal design of a hybrid distributed generation system. Power Engineering Conference (UPEC). Cluj-Napoca, 2014. pp. 1-6.

24. Akinyele D.O., Rayudu R.K., Nair N.K.C., Chakrabarti B. Decentralized energy generation for end-use applications: Economic, social and environmental benefits assessment. Innovative Smart Grid Technologies - Asia (ISGT Asia), IEEE. Kuala Lumpur, 2014. pp. 84-89.

25. Chernyakhovskaya Yu.V. The evolution of methodological approaches to the assessment of the cost of electricity. Analysis of foreign experience. Bulletin of the Ivanovo State Energy University, 2016, no. 4, pp. 56-68. In Rus.

26. Kovalev V.Z., Malgin G.V., Arkhipova O.V. Matematicheskoe modelirovanie elektrotekhnicheskikh kompleksov neftegazodobychi $v$ zadachakh energosberezheniya: monografiya [Mathematical modeling of electrical engineering complexes of oil and gas production in energy saving problems]. Khanty-Mansiysk, Department of Education and Science of the Khanty-Mansi Autonomous Okrug - Ugra, Ugra State University, 2008. 222 p.

27. Bolovin E.V., Glazyrin A.S. Method of identification of parameters of submersible asynchronous electric motors of installations of electric drive centrifugal pumps for oil production. Bulletin of the Tomsk Polytechnic University. Geo assets Engineering, 2017, vol. 328, no. 1, pp. 123-131. In Rus.

28. Kovalev V.Z., Shcherbakov A.G. Information system for identification of parameters of mathematical models of electrical systems. Control systems and information technology, 2009, vol. 35 , no. 1, pp. 57-59. In Rus.

29. Hairer E., Wanner G. Solving Ordinary Differential Equations II: Stiff and Differential-Algebraic Problems. Springer Series in Computational Mathematics, 1996, vol. 14, p. 614.

30. Gridin V.N., Ryzhov N.G., Anisimov V.I., Abukhazim M.M. Methods to Boost Performance Modeling of Dynamic Modes of Nonlinear Systems. Information Technologies, 2017, vol. 23, no. 11, pp. 796-802. In Rus.

31. Hall G. Modern Numerical Methods for Ordinary Differential Equations. Eds. G. Hall, J.M. Watt. Oxford, UK, Clarendon Press, $1976.312 \mathrm{p}$.

32. Tremblay 0., Dessaint L. Experimental validation of a battery dynamic model for EV application. World Electric Vehicle Journal, 2009, no. 3, pp. 1-10.

33. Thomachan A. Kattakayam, Srinivasan K. Lead acid batteries in solar refrigeration systems. Renewable Energy, 2004, vol. 29, pp. $1243-1250$.

34. Musgrove P. Wind Energy. Cambridge, Cambridge university press, 2010. $323 \mathrm{p}$.

35. Salas V., Olias E. Overview of the state of technique for PV inverters used in low voltage gridconnected PV systems: Inverters above 10kW. Renewable and Sustainable Energy Reviews, 2011, vol. 15 , pp. 1250-1257.

36. Hassaine L., Olias E., Quintero J. Overview of power inverter topologies and control structures for grid connected photovoltaic systems. Renewable and Sustainable Energy Reviews, 2014, vol. 30 , pp. 796-807.

37. Kost C., Shammugam S., Jülch V., Nguyen H.-T., Schlegl T. Levelized cost of electricity renewable energy technologies. Freiburg, Fraunhofer institute for solar energy systems ISE, 2018. 34 p.

38. Gear C.W. The Algorithm 407: DIFSUB for solution of ordinary differential equations. Communications of the ACM, 1971, vol. 14, no. 3, pp. 185-190.

Received: 17 December 2018.

\section{Information about the authors}

Olga V. Arkhipova, senior lecturer Yugra State University.

Vladimir Z. Kovalev, Dr. Sc., professor, Yugra State University.

Rustam N. Khamitov, Dr. Sc., professor, Omsk State Technical University. 